\title{
INFLUENCE OF THE VIEWING GEOMETRY WITHIN HYPERSPECTRAL IMAGES RETRIEVED FROM UAV SNAPSHOT CAMERAS
}

\author{
Helge Aasen \\ GIS and RS Research Group, Institute of Geography, University of Cologne, 50923 Cologne, Germany (helge.aasen@uni-koeln.de)
}

KEY WORDS: BRDF, angular effects, vegetation index, barley, image-frame camera

\begin{abstract}
:
Hyperspectral data has great potential for vegetation parameter retrieval. However, due to angular effects resulting from different sunsurface-sensor geometries, objects might appear differently depending on the position of an object within the field of view of a sensor. Recently, lightweight snapshot cameras have been introduced, which capture hyperspectral information in two spatial and one spectral dimension and can be mounted on unmanned aerial vehicles.

This study investigates the influence of the different viewing geometries within an image on the apparent hyperspectral reflection retrieved by these sensors. Additionally, it is evaluated how hyperspectral vegetation indices like the NDVI are effected by the angular effects within a single image and if the viewing geometry influences the apparent heterogeneity with an area of interest. The study is carried out for a barley canopy at booting stage.

The results show significant influences of the position of the area of interest within the image. The red region of the spectrum is more influenced by the position than the near infrared. The ability of the NDVI to compensate these effects was limited to the capturing positions close to nadir. The apparent heterogeneity of the area of interest is the highest close to a nadir.
\end{abstract}

\section{INTRODUCTION}

Hyperspectral data has been shown to have great potential for the estimation biophysical and biochemical properties of vegetation (Aasen et al., 2014b; Thenkabail et al., 2012; Tilly et al., 2015). Recently, new types of lightweight hyperspectral cameras have been introduced, which can be mounted on unmanned aerial vehicles (UAVs). Image-frame and snapshot cameras like the Cubert UHD-185 (Aasen et al., 2015; Bareth et al., 2015), the Rikola FPI (Honkavaara et al., 2013) or the imec SM series (Constantin et al., 2015) record hyperspectral data in two spatial and one spectral dimension.

Since airborne hyperspectral sensors are commonly passive systems, they depend on the illumination of the sun. Besides, within the field of view (FOV) of imaging systems, the surface is seen from different angles depending on the pixel position within the detector array. Thus, the sun-surface-sensor geometry is unique for every pixel and depends on the suns position and the orientation of the sensor (Schaepman-Strub et al., 2006). Additionally, vegetation acts like an anisotropic surface and the appearance of a vegetation canopy might differ when seen form a different perspective (Jones and Vaughan, 2010). Thus, also the apparent reflectance within an area of interest (AOI) perceived by a hyperspectral sensing system may differ depending on the viewing geometry, respectively the position of the AOI within the image.

Such angular effects have been shown to influence the retrieval of plant parameters (Burkart et al., 2015; Verrelst et al., 2008) and data products such as hyperspectral digital surface models (Aasen and Bolten, in review). Thus, approaches have been developed which try to compensate these effects (Schläpfer et al., 2015). On the other hand, it has been shown that multispectral multi-angular observations can be used to retrieve additional information about vegetation canopies (Koukal et al., 2014). The scope of this study is to investigate the influence of the different viewing geometries within one hyperspectral image retrieved by a lightweight UAV snapshot camera. Additionally, it is evaluated if hyperspectral vegetation indices (VIs), like the NDVI, can compensate the angular effects and if the viewing geometry influences the apparent heterogeneity within an AOI.

\section{BACKGROUND}

\subsection{Snapshot camera based remote sensing}

Hyperspectral bush-broom systems are widely known in the remote sensing community. They record spectral information with one spatial and one spectral dimension. Commonly, every image line is recorded perpendicular to the longitudinal axis of the carrier platform (e.g. aircraft) or flight direction (e.g. satellite). Their FOV is divided into several pixels, each with an own instantaneous field of view (IFOV). Thus, the viewing direction of each pixel is slightly different (Jones and Vaughan, 2010). To minimize angular effects resulting from different sunsurface-sensor geometries, flight lines are usually planned along the solar principle plain.

Snapshot cameras record spectral information in two spatial and one spectral dimension. Compared to bush-broom systems, one more spatial dimension has to be taken into account. In the following, the spatial dimension perpendicular to the flight direction will be referred to as across-track and the dimension in parallel to the flight direction will be referred to as along-track. The additional spatial dimension brings additional complexity into the data derived by these systems (Aasen et al., 2015, 2014a).

\subsection{Angular scattering effects of vegetation}

Plant canopies show an asymmetrical scattering behavior. Their structural and optical properties, multiple scattering within the canopy, mutual shadowing, transmission, reflectance and absorption influence the way light is absorbed and reflected (Gatebe, 2003). In this study, barley vegetation at booting stage with an almost closed canopy was present within the AOI. Cereal canopies show a lower forward scattering than backward scattering due to casting shadows. Additionally, they show a hotspot feature directed towards the direct incidence irradiance, which is more pronounced in the visible region (VIS) than in the near infrared region (NIR). Additionally, at nadir they show a local maximum in the VIS and an local minimum at the NIR (Gerstl and Simmer, 1986; Küster et al., 2014). 


\section{METHODS}

\subsection{UAV hyperspectral sensing system}

In this study the hyperspectral snapshot camera UHD 185-Firefly (UHD; Cubert GmbH, www.cubert-gmbh.de) was used. The camera records an image cube (IC) with 138 spectral bands from 450 to $950 \mathrm{~nm}$ with a spatial resolution of 50 by 50 pixels with each integration. The FWHM max ranges from approximately 5 to $25 \mathrm{~nm}$. Simultaneously to the IC, a grayscale image with 1000 by 990 pixels is recorded. The field of view (FOV) of the camera is approx. $20^{\circ}$ in across- and along-track direction. This results in a spatial resolution of the hyperspectral information of approx. $20 \mathrm{~cm}$ at $30 \mathrm{~m}$ above ground. The grayscale image has a ground resolution of approx. $1 \mathrm{~cm}$. The camera is connected to a single board computer (Pokini Z, http://www.pokini.de/), which controls the camera and records the data. The system records 0.6 frames per second.

The camera is mounted on a gimbal (MK HiSight SLR2 gimbal, MikroKopter, www.mikrokopter.de) to ensure a nadir viewing direction. The camera and the single board computer are mounted on a UAV (OktoXL 2, MikroKopter, www.mikrokopter.de). Altogether, the sensing system weight is about $5 \mathrm{~kg}$ and the flight time is about 20 minutes depending on the wind conditions.

\subsection{Angular properties of the image cubes}

The theoretical framework to describe angular effects of a surface is the bidirectional reflectance distribution function (BRDF) (Nicodemus et al., 1977). Depending on the composition of the incident irradiance and the properties of the sensing system different cases are distinguished (Schaepman-Strub et al., 2006). The FOV of the UHD is $20^{\circ}$ and is divided into 50 pixels in every spatial dimension. Thus, the instantaneous field of view (IFOV) of every pixel is about $0.4^{\circ}$. By definition, directional measurements can only be derived by sensing systems with an infinitesimal small solid view angle. However, in reality infinitesimally small IFOVs do not exist (Schaepman-Strub et al., 2006). Because of the small viewing angle per pixel of the UHD, the measurements can be regarded as directional. The measurements were transformed to reflectance factors relative to a white reference panel (Zenith Light, www.sphereoptics.de) measured before take-off with the same viewing geometry as the subsequent measurements of the vegetation.

The field study was carried out at the research station Campus Klein-Altendorf close to the city of Bonn, Germany, on the $20^{\text {th }}$ May 2014 at 1:30 pm local time. The sun had an elevation of $59.35^{\circ}$ and an azimuth angle of $180.42^{\circ}$. The experiment was carried out on a sunny day with no clouds visible. Thus, the main contributor of incident light was the sun. Additionally, as usual for field conditions, the hemisphere contributed diffuse irradiation. Thus, the incident light conditions can be described as hemispherical and the measurements represent hemispherical directional reflectance factors.

The flight direction was about north south, resulting in an alongtrack direction parallel to the suns principle plain. Because of the orientation of the ICs, the IFOV of pixels with a low along-track pixel position had a sun-angle of approx. $20^{\circ}$, while the IFOV of pixels with a high along-track position had a sun-angle of approx. $40^{\circ}$.

\subsection{Image cube pre-processing and orthorectification}

The ICs preparation was done according to Aasen et al. (2015) and included i) converting the DN to reflectance, ii) merging the high resolution grayscale image to the low resolution spectral data and iii) attaching a pixel position band to the composed IC.
From the raw DN values the dark-current was subtracted and the ICs were calibrated to reflectance by dividing them through a measurement of a white reference panel, captured before the flight. Then, spectral bands were merged with the corresponding grayscale image and converted to the same spatial resolution with the help of a nearest neighbor interpolation. Since the grayscale image has a resolution of $1000 \times 990$ pixels, the value of one hyperspectral pixel was assigned to about 400 grayscale pixels. Additionally, a pixel id was assign to each hyperspectral pixel to trace the pixel position within the original IC.

All ICs were imported into Photoscan (version 1.1.6; Agisoft, www.agisoft.com), aligned and georeferenced with ground control points measured with a D-GPS with an accuracy of approx. $1 \mathrm{~cm}$. Then, relevant ICs were selected and exported with the help of a python script. The export mode was chosen such that pixel value interpolation was prevented. During that process Photoscan not only georeferenced but also orthorectified the individual ICs.

\subsection{Pixel data base generation}

Each orthorectified IC was imported into MATLAB (version R2014a, www.mathworks.com). During the fieldwork the plot corners were measured with a D-GPS. From these corners an AOI was created for the plot. To exclude border effects, a buffer of $0.8 \mathrm{~m}$ from the edges was used. The AOI was stored as a shapefile. For each IC, the spectral information together with the original pixel position of all pixels within the AOI were extracted. Due to the orthorectification process, some pixels had missing or erroneous values. These values were filtered by omitting missing $(\mathrm{NaN})$ values and applying the median to all pixels with the same pixel position. The resulting value represented the real hyperspectral value for the specific pixel position with the particular IC. This value was stored to a pixel database (PIX DB). The procedure was applied to all ICs, which contained pixels within the AOI and is summarized in Figure 1. The resulting PIX DB contained the spectral information of all pixels of all ICs together with their original pixel position within the IC.

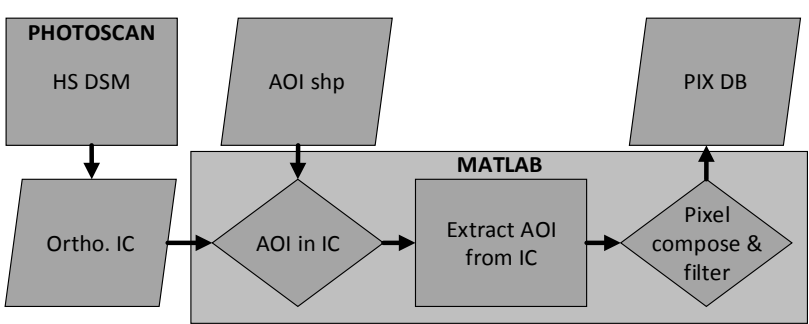

Figure 1 Pixel database (PIX DB) generation for an area of interest (AOI) from orthorectrified image cubes (ortho IC).

\section{RESULTS AND DISCUSSION}

\subsection{Pixel extraction}

Figure 3 shows the reflectance at $670 \mathrm{~nm}$ of the orthorectified IC2 as an overlay on an RGB image of one plot. The AOI, which was extracted for the creation of PIX DB, is highlighted with a black frame. Within the AOI a pattern is visible. To the southwest of the plot, a triangle with high reflectance is visible. In the center of the plot, a part with lower reflectance can be identified. To the east and northeast, one pixel with noticeable increased reflectance can be identified.

The AOI investigated in this study was visible in 9 ICs. From these, we selected 6 ICs which covered the entire AOI (for IC1 some pixels are missing to the north of the AOI), did not overlap with regard to the pixel position and were captured within the 
same UAV overpass. From the PIX DB the spectral information for the pixels of these ICs were selected for the analysis.

Figure 2 shows a visualization of these pixels at their along-track (x-axis) and across-track (y-axis) pixel position. Since each IC is captured from a different location and is orthorectified in a different way, the shapes of the extracted pixel slightly differ for each IC. As already mentioned, for IC1 some pixels to the left side of the plot are missing since the AOI slightly exceeded the footprint of the IC.

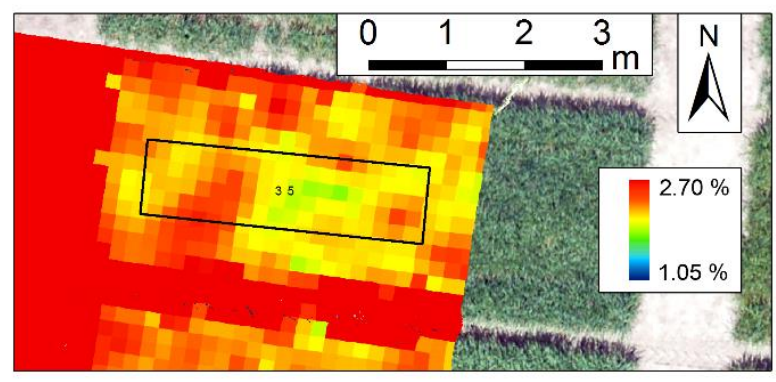

Figure 3 Image cube 2 (IC2) with the reflectance at $670 \mathrm{~nm}$ as an overlay on a RGB image.
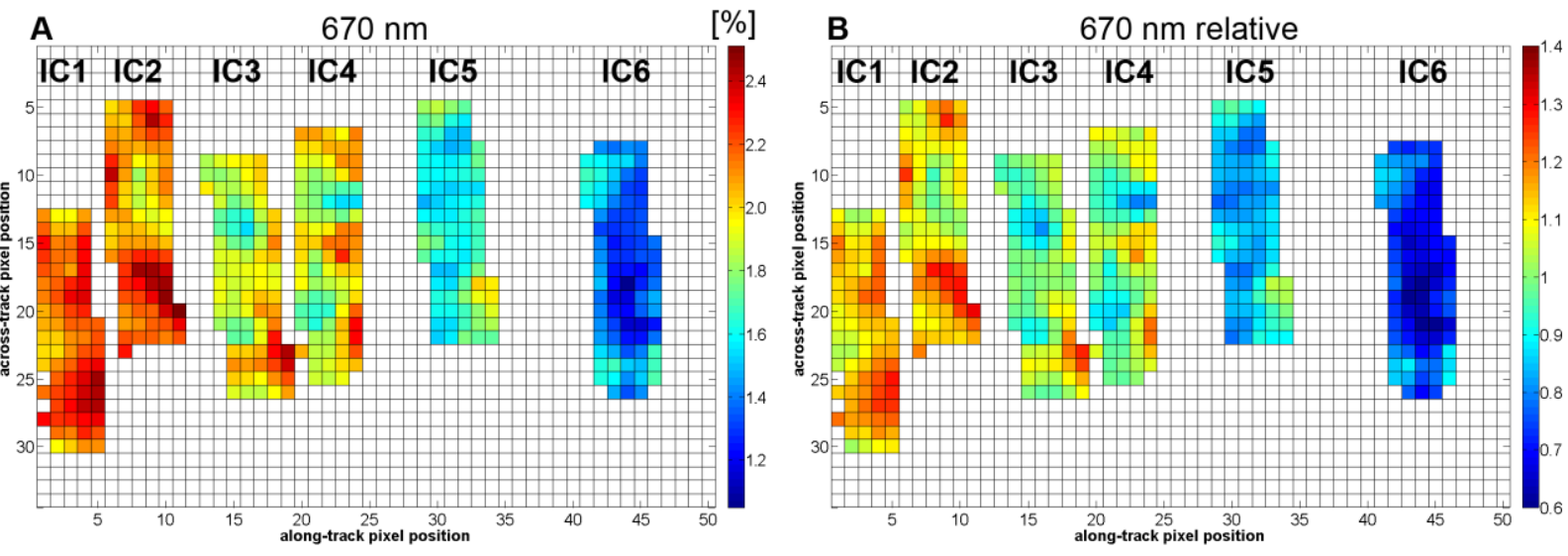

C

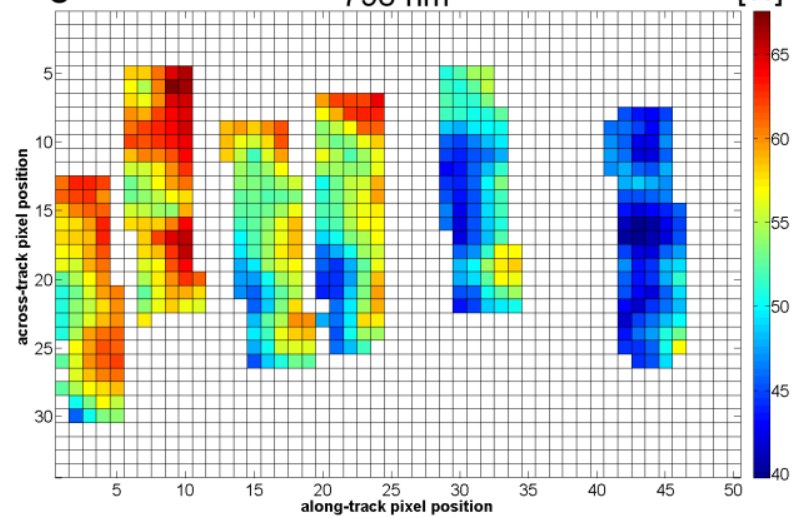

E

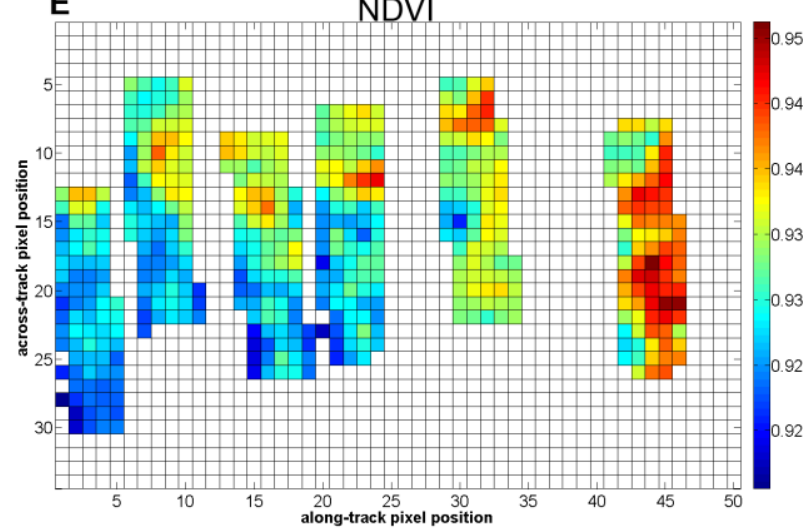

D

$798 \mathrm{~nm}$ relative

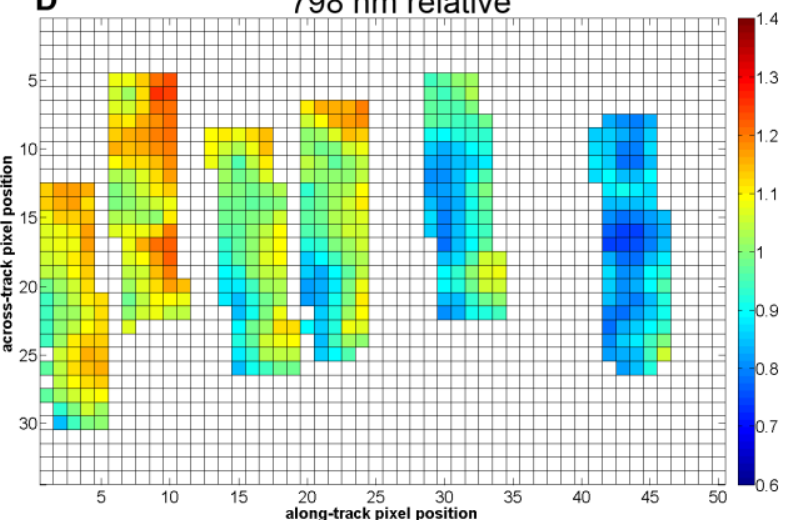

$\mathbf{F}$

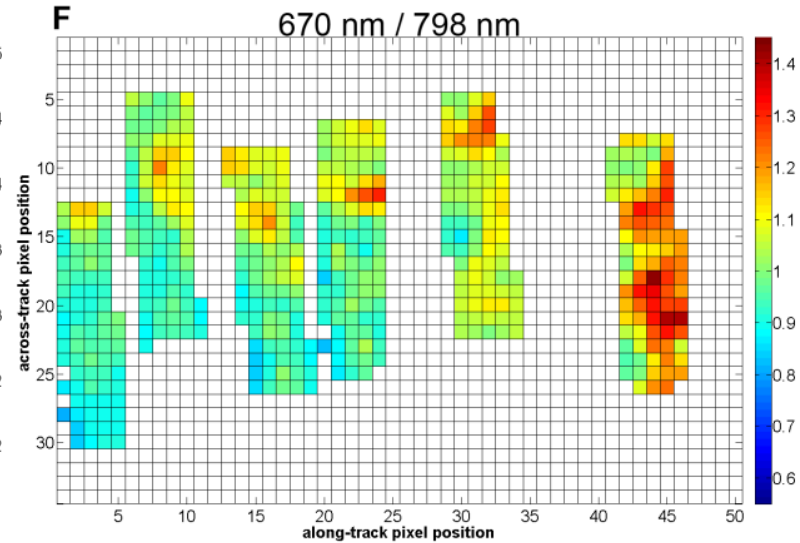

Figure 2 Visualization of the spectral information for the available pixel position within the pixel database for image cube (IC) 1 to 6 at $670 \mathrm{~nm}$ and $798 \mathrm{~nm}$ as absolute reflectance values (A,C) and relative values (B,D). Additionally, the NDVI (E) and the ratio of the relative reflectance at $670 \mathrm{~nm}$ and $798 \mathrm{~nm}(\mathrm{~F})$ is shown. 


\subsection{Influence on the apparent reflection}

Figure 2-A and $\mathrm{C}$ show the apparent reflection at $670 \mathrm{~nm}$ and $798 \mathrm{~nm}$, respectively. In both wavelength, the reflection decreases with increasing along-track pixel position. At $670 \mathrm{~nm}$, the reflection of IC1 ranges from $1.95 \%$ to $2.45 \%$. In IC6, the reflection rages from $1.05 \%$ to $1.71 \%$. The apparent average reflection of the plot decreases by $0.79 \%$ reflectance from IC1 $(2.19 \%)$ to IC6 $(1.40 \%)$, which is more than the in plot heterogeneity and about one third of the maximum average reflection. At $798 \mathrm{~nm}$, the reflection in IC1 ranges from $45.66 \%$ to $63.04 \%$. In IC6, it ranges from $39.76 \%$ to $57.00 \%$. The average decreases by $12.27 \%$ reflectance from $57.59 \%$ to $45.32 \%$ and thus, by about one fifth of the maximum reflection. Figure 2-B and D show the relative reflection for both wavelength normalized by the median of all pixels from all ICs. At $670 \mathrm{~nm}$, the relative reflection ranges from 0.55 to 1.31 . At $798 \mathrm{~nm}$, the relative reflectance ranges from 0.74 to 1.26 . Figure 4 shows the relative mean reflectance per IC as a function of the average across-track pixel position of each IC.

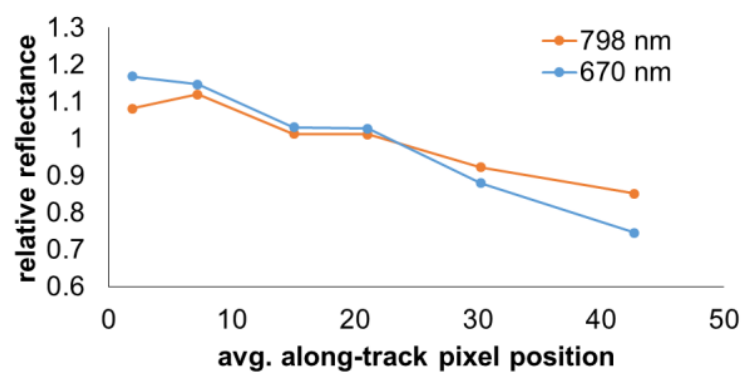

Figure 4 Average reflectance per IC for $670 \mathrm{~nm}$ and $798 \mathrm{~nm}$ in relation to the average (avg.) along-track pixel position

In alignment with the visual impression of Figure 2, the average reflection of both wavelength decreases with increasing across track position. Additionally, the average change is higher at $670 \mathrm{~nm}(42 \%)$ than at $798 \mathrm{~nm}(23 \%)$. For IC3 and IC4 hardly any difference is observable. The average across-track pixel position of IC3 and IC4 is 15px and 21px an thus, within only 5px from the nadir viewing direction The relative reflectance of these two ICs only differ by less than 0.004 for both wavelength.

Pixels with a lower along-track position have a more acute angle to the incident irradiation from the sun than pixels with a higher along-track pixel position. Thus, they are closer to the hotspot feature apparent for anisotropic surfaces and show a higher reflectance. These results aligns with other studies, which also found that the relative influence of the view angle on the apparent reflection of vegetation in the VIS is considerable higher than in the NIR, were light is scattered within the canopy (Burkart et al., 2015; Küster et al., 2014; Sandmeier et al., 1999; Schläpfer et al., 2015).

\subsection{Influence on vegetation indices}

In Figure 2-E the NDVI is shown for each pixel of the ICs. In IC1 the NDVI values range from 0.92 to 0.94 . In IC6 they range from 0.93 to 0.95 . Thus, controversy to the individual wavelength, the NDVI values are increasing with increasing along-track pixel position. This is a result of the absolute larger increase in the NIR than in the red and thus, an increase of the denominator in the NDVI formula. Figure 5 shows the average, minimum and maximum NDVI values of the ICs as a function of the average along-track pixel position.

The graphs complement the visual impression and confirm that the NDVI is increasing with along-track pixel position. For IC2 to IC4, only minor changes of the average NDVI are observed.
For these ICs, respectively these along-track pixel positions, the relative reflectance of the red and NIR show similar values (Figure 2). Thus, the change in reflectance due to the different geometries can be compensated. For the other ICs, the relative reflectance of the two wavelength develop differently which affects the NDVI values.

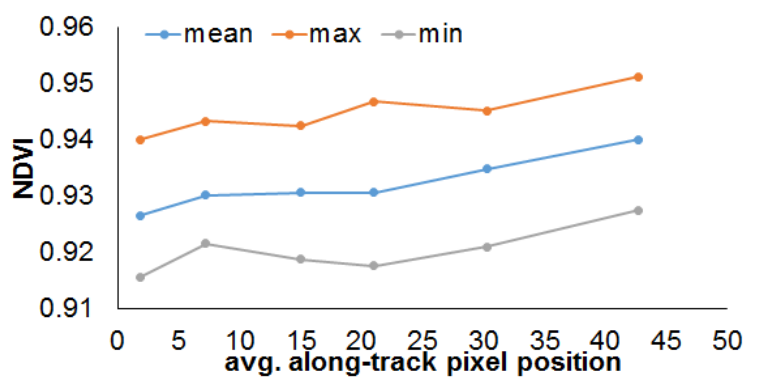

Figure 5 Average (mean), minimum (min) and maximum (max) NDVI values of the image cubes as a function of the average along-track pixel position.

\subsection{Angular effects on apparent heterogeneity}

In Figure 2-A and $\mathrm{C}$ heterogeneity within the plot is visible. The areas identified in Figure 3 can also be identified in most ICs captured from the other perspectives. However, the pattern is more pronounced at $670 \mathrm{~nm}$ than at $798 \mathrm{~nm}$. Additionally, $670 \mathrm{~nm}$ is more sensitive to the perspective than $798 \mathrm{~nm}$ (Figure $2-\mathrm{B}, \mathrm{D})$. The visible heterogeneity with the AOI differs between the ICs. Figure 6 shows the coefficient of variation $(\mathrm{CV})$ for IC1 to 6 at $670 \mathrm{~nm}$ and $798 \mathrm{~nm}$ in relationship to the average acrosstrack pixel position of the plot.

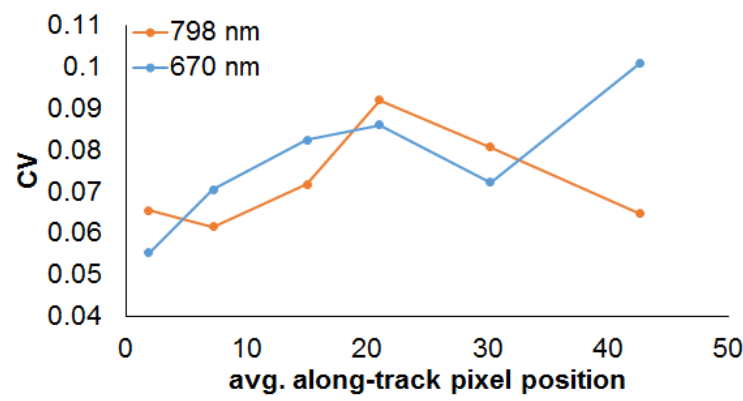

Figure 6 Coefficient of variation (CV) for image cubes 1 to 6 at $670 \mathrm{~nm}$ and $798 \mathrm{~nm}$ in relationship to the average across-track pixel position of the plot.

Overall, the $\mathrm{CV}$ and thus the apparent variability within the AOI increases towards the nadir perspective and decreases towards lower and higher pixel positions. Only at $670 \mathrm{~nm}$ the CV steeply increases in IC6.

In-plot heterogeneity can result from various reasons. Different sowing densities or varmints might have influenced the growth Additionally, the area to the north and south of the AOI is close to a sowing track of the tractor, which might have influenced the soil compression and water and light availability of the plants. The different effects influence vegetation greenness and soil visibility and thus, the reflectance in different wavelength. The generally highest variability seen in the close to nadir viewing direction. This might result from a higher soil fraction seen from this perspective. However, more research is needed to identify how the different perspectives influence what is seen within an AOI. Nevertheless, this could indicate that viewing geometries closer to nadir are beneficial to detect differences in dense vegetation 


\section{CONCLUSION}

This study evaluated the influence of angular effects within hyperspectral images of snapshot cameras. Significant differences of the apparent reflectance were found with changing along-track position, respectively along the suns principle plain. These changes were more pronounced in the red region than in the near infrared region. The ability of the NDVI to compensate these effects was limited to the capturing positions close to nadir. The apparent heterogeneity increased strongly towards the nadir viewing direction which could indicate that nadir viewing geometries are beneficial for detecting differences in dense cereal canopies. Overall, the results highlight the influence of the viewing geometry on the apparent reflectance. Thus, further research is needed to understand, compensate or exploit this phenomenon. Since UAV snapshot cameras record hyperspectral data in two spatial dimensions they represents an optimal tool for such investigations.

\section{ACKNOWLEDGEMENTS}

I am very grateful to Andreas Bolten for his technical support with and piloting of the UAV. Helge Aasen was supported by the Grant No. GSGS-2015B-F01 of the Graduate School of Geosciences, University of Cologne during the preparation of this manuscript. The field experiment was established during the CROP.SENSe.net project in context of the Ziel 2-Programms NRW 2007-2013 "Regionale Wettbewerbsfähigkeit und Beschäftigung", financially supported by the Ministry for Innovation, Science and Research (MIWF) of the state North Rhine Westphalia (NRW) and European Union Funds for regional development (EFRE) (005-1103-0018).

\section{REFERENCES}

Aasen, H., Bendig, J., Bolten, A., Bennertz, S., Willkomm, M., Bareth, G., 2014a. Introduction and preliminary results of a calibration for full-frame hyperspectral cameras to monitor agricultural crops with UAVs, in: ISPRS International Archives of the Photogrammetry, Remote Sensing and Spatial Information Sciences. pp. 1-8.

Aasen, H., Bolten, A., in review. Multi-temporal monitoring of agricultural crops with high-resolution 3D hyperspectral digital surface models in comparison with ground observations. Remote Sens. Environ.

Aasen, H., Burkart, A., Bolten, A., Bareth, G., 2015. Generating 3D hyperspectral information with lightweight UAV snapshot cameras for vegetation monitoring: From camera calibration to quality assurance. ISPRS J. Photogramm. Remote Sens. 108, 245-259.

Aasen, H., Gnyp, M.L., Miao, Y., Bareth, G., 2014b. Automated Hyperspectral Vegetation Index Retrieval from Multiple Correlation Matrices with HyperCor. Photogramm. Eng. Remote Sens. 80, 785-795.

Bareth, G., Aasen, H., Bendig, J., Gnyp, M.L., Bolten, A., Jung, A., Michels, R., Soukkamäki, J., 2015. Low-weight and UAV-based Hyperspectral Full-frame Cameras for Monitoring Crops: Spectral Comparison with Portable Spectroradiometer Measurements. Photogramm. Fernerkund. - Geoinformation 2015, 69-79.

Burkart, A., Aasen, H., Alonso, L., Menz, G., Bareth, G., Rascher, U., 2015. Angular Dependency of Hyperspectral Measurements over Wheat Characterized by a Novel UAV Based Goniometer. Remote Sens. 7, 725-746.
Constantin, D., Rehak, M., Akhtman, Y., Liebisch, F., 2015. Detection of crop properties by means of hyperspectral remote sensing from a micro UAV, in: 9th EARSeL SIG Imaging Spectroscopy Workshop.

Gatebe, C.K., 2003. Airborne spectral measurements of surfaceatmosphere anisotropy for several surfaces and ecosystems over southern Africa. J. Geophys. Res. 108.

Gerstl, S.A.W., Simmer, C., 1986. Radiation physics and modelling for off-nadir satellite-sensing of nonLambertian surfaces. Remote Sens. Environ. 20, 1-29.

Honkavaara, E., Saari, H., Kaivosoja, J., Pölönen, I., Hakala, T., Litkey, P., Mäkynen, J., Pesonen, L., 2013. Processing and Assessment of Spectrometric, Stereoscopic Imagery Collected Using a Lightweight UAV Spectral Camera for Precision Agriculture. Remote Sens. 5, 5006-5039.

Jones, H.G., Vaughan, R.A., 2010. Remote sensing of vegetation: principles, techniques, and applications. Oxford University Press, Oxford; New York.

Koukal, T., Atzberger, C., Schneider, W., 2014. Evaluation of semi-empirical BRDF models inverted against multiangle data from a digital airborne frame camera for enhancing forest type classification. Remote Sens. Environ. 151, 27-43.

Küster, T., Spengler, D., Barczi, J.-F., Segl, K., Hostert, P., Kaufmann, H., 2014. Simulation of Multitemporal and Hyperspectral Vegetation Canopy Bidirectional Reflectance Using Detailed Virtual 3-D Canopy Models. IEEE Trans. Geosci. Remote Sens. 52, 20962108.

Nicodemus, F.E., Richmond, J.C., Hsia, J.J., Ginsberg, I.W., Limperis, T., 1977. Geometrical considerations and nomenclature for reflectance. National Bureau of Standards, Washington DC, p. 67.

Sandmeier, S.R., Itten, K., others, 1999. A field goniometer system (FIGOS) for acquisition of hyperspectral BRDF data. Geosci. Remote Sens. IEEE Trans. On 37, 978-986.

Schaepman-Strub, G., Schaepman, M.E., Painter, T.H., Dangel, S., Martonchik, J.V., 2006. Reflectance quantities in optical remote sensing - definitions and case studies. Remote Sens. Environ. 103, 27-42. 2

Schläpfer, D., Richter, R., Feingersh, T., 2015. Operational BRDF Effects Correction for Wide-Field-of-View Optical Scanners (BREFCOR). IEEE Trans. Geosci. Remote Sens. 53, 1855-1864.

Thenkabail, P.S., Lyon, J.G., Huete, A. (Eds.), 2012. Hyperspectral remote sensing of vegetation. CRC Press, Boca Raton, FL.

Tilly, N., Aasen, H., Bareth, G., 2015. Fusion of Plant Height and Vegetation Indices for the Estimation of Barley Biomass. Remote Sens. 7, 11449-11480.

Verrelst, J., Schaepman, M.E., Koetz, B., Kneubühler, M., 2008. Angular sensitivity analysis of vegetation indices derived from CHRIS/PROBA data. Remote Sens. Environ. 112, 2341-2353. 